Original paper

\title{
Impact of liver-directed therapy and non-therapy on the waiting time list of patient candidates for liver transplantation: retrospective survival analysis
}

\author{
Maria del Pilar Bayona Molano', Lorena Garza², Genaro Selvaggi², Jay Vasani², Juan Carlos Barrera Gutierrez ${ }^{3}$, \\ Jason Salsamendi², Shivank Bhatia², Leopoldo Arosemena \\ ${ }^{1}$ Mallinckrodt Institute, Barnes Jewish Hospital, St. Louis, USA \\ 2University of Miami Miller School of Medicine, Miami, USA \\ ${ }^{3}$ Kent State University, College of Public Health, Kent, USA
}

\begin{abstract}
Aim of the study: To determine whether liver-directed therapies (LDT) and no therapy affect waiting list times for liver transplant candidates from a single center.

Material and methods: This retrospective study included patients $>12$ years of age diagnosed with hepatocelIular carcinoma between January 2014 and June 2019 and followed until the date of transplant, date of delisting, loss to follow-up, or date of death. Waiting list time and associated factors were analyzed using Kaplan-Meier and Cox proportional-hazards methods.

Results: A total of 181 patients met the selection criteria. The mean age was 60 years with standard deviation (SD) of 7.8 years. Sixty-six percent underwent transplant, and $64 \%$ were classified within the Milan criteria. Men had a lower median waiting list time than women (191 days vs. 236 days, $p=0.0093$ ). The overall median survival time or time to transplant for $50 \%$ of the population was 218 days ( $95 \% \mathrm{Cl}$ : 195-235). Men displayed a 3.1-fold (95\% Cl: 1.5-6.2) higher probability of transplantation than women ( $p=0.002)$. Patients who received no therapy had a 5 -fold higher probability of undergoing transplantation than patients under arterial LDT (HR [95\% Cl]: $5[1.2,20], p=0.02$ ). Patients under combined LDT displayed a $70 \%$ reduced probability of transplantation compared to patients who received arterial LDTs $(p=0.0009)$.

Conclusions: LDT was associated with a prolonged stay on the transplant list, likely due to the presence of an aggressive liver tumor. However, LDTs allow the patient to remain active on the liver transplant list, increasing their chances of undergoing transplantation.
\end{abstract}

Key words: hepatocellular carcinoma, liver transplant, liver-directed therapies, waiting list time.

Address for correspondence:

Dr. Maria del Pilar Bayona Molano, Mallinckrodt Institute, Barnes Jewish Hospital, St. Louis, USA, e-mail: cienimagenes@hotmail.com

\section{Introduction}

Liver transplantation is associated with superior survival compared to liver resection in patients with solitary hepatocellular carcinoma (HCC) that meet the United Network for Organ Sharing (UNOS) criteria (single lesion, diameter $\leq 50 \mathrm{~mm}$, no vascular invasion, no extrahepatic metastasis) [1], but transplantation is restricted due to limited availability of graft donors. Approximately $50 \%$ of people on the liver transplant waiting list will receive an organ within 5 years [2]. Once a patient is considered a candidate for a liver transplant, their waiting list position depends on several variables, including disease severity, donor availability, transplant region, and matching factors for the specific organ [2].

In 2002, UNOS adopted the Model for End-stage Liver Disease (MELD) score, which predicts the 
3-month survival rate in patients with end-stage liver disease, as a criterion for liver allocation to prioritize patients awaiting liver transplant (LT) [3]. After adoption of the MELD score, the average waiting time for transplant candidates decreased from 1314 days or 3.6 years in 1999-2000 to 457 days or 1.25 years in 2003-2004 [4]. According to the Organ Procurement and Transplantation Network (OPTN) and UNOS, the median national waiting time in 2006 was 321 days [5]. Between July 1, 2009 and December 31, 2014, the median national time to transplant patients registered on the waitlist was 16 months (480 days), as reported by the Scientific Registry of Transplant Recipients (SRPR). However, the waiting time varied by OPTN region; for candidates living in region 7 , the median time to transplant was approximately 14 months (420 days) [6].

Given the imperfect correlation between MELD score and waiting list outcomes, the OPTN established a system of exception points that better reflect the patient's prognosis and general condition in order to improve the patient selection approach [7]. In 2015, OPTN implemented a policy in which "candidates will receive the additional priority as long as they continue to meet initial eligibility criteria". This new phrasing is intended to capture patients who exceed initial eligibility criteria, progressing beyond $\mathrm{T} 2$ criteria (definition of T2 HCC lesions: 1) one lesion greater than or equal to $2 \mathrm{~cm}$ and less than or equal to $5 \mathrm{~cm}$ in size; 2) two or three lesions greater than or equal to $1 \mathrm{~cm}$ and less than or equal to $3 \mathrm{~cm}$ in size) [8].

Liver-directed therapies (LDTs) are minimally invasive interventions that not only provide treatment but also function as bridging or down-staging therapies for patients pursuing a liver transplant. The purpose of providing an LDT prior to liver transplant is to control tumor growth, reduce dropout in patients within the Milan criteria while awaiting liver transplantation, and decrease the tumor burden in patients outside the Milan criteria so they can become LT candidates. The overall risk of dropout in HCC patients waiting for LT and neoadjuvant therapies has been reported to be approximately $11 \%$ and $57 \%$ at 6 and 12 months, respectively [9]. However, a lower incidence of dropout $(0-25 \%$ in one year) was recently reported in another study [10].

As recommended by an international consensus and multiple societies, including the American Association for the Study of Liver Diseases (AASLD), if their transplant waiting time is estimated to be longer than 6 months, a patient in stage II or T2 of the TNM Classification of Malignant Tumors (TNM) will require bridging LDT, including ablations and trans-arterial embolization, to avoid progression [11]. Additionally, LDT or neoadjuvant treatments in patients beyond the Milan criteria (down-staging) are considered important alternatives of treatment for T3 (TNM) tumors. LDTs are used to transition these tumors to stage T2 (TNM) to allow the patient to qualify as a transplant candidate. Combined LDTs have been described as potentially more effective alternatives to single LDTs [12]. Selection of the LDT depends on several guidelines, including the Barcelona Clinic Liver Cancer Classification (BCLC) recommendations [13].

Despite our knowledge of the efficacy of LDT for HCC, the impact of LDTs on the transplant waiting list remain unclear. This study aimed to determine whether use of LDTs and no therapy affect the waiting list time for liver transplantation candidates from a single center located in region 3 of the OPTN.

\section{Material and methods}

This study obtained Institutional Review Board (IRB) approval. This retrospective analysis reviewed patients from a single transplant center who were diagnosed with HCC between January 2014 and June 2019 and who were enrolled in the UNOS transplant waiting list. All participants provided informed consent before each diagnostic procedure of HCC, LDTs, and LT. Selection criteria included patients $>12$ years of age with diagnoses of HCC, and candidates for LT according to criteria (tumor size and number of nodules) defined by the selection committee. All data were sourced from patient clinical records. Patients' age was within the range of 37 to 81 years, and the mean age at HCC diagnosis was 60 (standard deviation [SD] = 7.8) years. Men were affected at a 3-fold higher rate than women ( $77 \%$ vs. $23 \%$ ). The study population was $53 \%$ Hispanic, $41 \%$ non-Hispanic Whites, and $6 \%$ other races, including African Americans and Asians. See Table 1 for more information about the study population.

\section{Terms and measures}

The event "transplant" was used as the dependent variable to indicate whether the patient did or did not undergo transplantation. The time to event or "waiting list time" was considered the time in days between the date when the patient was listed for transplant and the date when the patient underwent transplantation or the date when the patient was delisted because of death, disease progression, or loss to follow-up.

As independent variables, we selected age at diagnosis of HCC, sex, ethnicity, and comorbidities such 
Table 1. Characteristics of liver transplant candidates, and median days on the waiting list, $N=181$

\begin{tabular}{lccccc}
\hline Variables & $n$ & \% or SD & $95 \%$ Cl & $\begin{array}{c}\text { Median } \\
\text { days WL }\end{array}$ & -value \\
\hline Age (mean) & 60 & 7.8 & & & \\
\hline Sex & & & & & \\
\hline Male & 121 & 77 & $70-84$ & 191 & 0.0093 \\
\hline Female & 36 & 23 & $16-30$ & 236 & \\
\hline Ethnicity & & & & & \\
\hline Hispanics & 83 & 53 & $45-61$ & 204 & 0.7421 \\
\hline Non-Hispanic & 64 & 41 & $33-49$ & 206 & \\
Whites & & & & & \\
\hline Others & 10 & 6 & $3-10$ & 192 & \\
\hline Etiology & & & & & \\
\hline Viral & 65 & 36 & $29-43$ & 211 & 0.6148 \\
\hline NASH & 28 & 15 & $10-21$ & 208 & \\
\hline Alcohol & 25 & 14 & $9-19$ & 205 & \\
\hline Unspecified & 56 & 31 & $24-38$ & 192 & \\
\hline Others & 7 & 4 & $1-7$ & 263 & \\
\hline Comorbidities & & & & & \\
\hline One & 71 & 71 & $62-80$ & 213 & 0.243 \\
\hline Two & 22 & 22 & $14-30$ & 218 & \\
\hline Three & 7 & 7 & $2-12$ & 94 & \\
\hline TNM classification & & & & & \\
\hline TON0M0 & 2 & 1 & $0-3$ & & \\
\hline T1N0M0 & 20 & 13 & $8-18$ & 132 & 0.1827 \\
\hline T2N0M0 & 100 & 64 & $56-72$ & 218 & \\
\hline T3N0M0 & 34 & 22 & $15-28$ & 194 & \\
\hline Milan criteria & & & & & \\
\hline Criteria & 100 & 64 & $56-72$ & 218 & \\
\hline
\end{tabular}

\begin{tabular}{|c|c|c|c|c|c|}
\hline Variables & $n$ & $\%$ or SD & $95 \% \mathrm{Cl}$ & $\begin{array}{l}\text { Median } \\
\text { days WL }\end{array}$ & $P$-value \\
\hline $\begin{array}{l}\text { Outside of } \\
\text { Milan criteria }\end{array}$ & 56 & 36 & $28-43$ & 189 & \\
\hline \multicolumn{6}{|l|}{ Therapy } \\
\hline Intra-arterial & 74 & 51 & $42-59$ & 210 & 0.058 \\
\hline Ablations & 16 & 11 & $6-16$ & 213 & \\
\hline $\begin{array}{l}\text { Combined } \\
\text { therapy }\end{array}$ & 39 & 27 & $20-34$ & 237 & \\
\hline No therapy & 16 & 11 & $6-16$ & 116 & \\
\hline \multicolumn{6}{|c|}{ Number of cycles } \\
\hline Single cycle & 52 & 36 & $28-44$ & 206 & 0.058 \\
\hline $\begin{array}{l}\text { Multiple } \\
\text { cycles }\end{array}$ & 38 & 26 & $20-33$ & 213 & \\
\hline $\begin{array}{l}\text { Combined } \\
\text { therapy }\end{array}$ & 39 & 27 & $20-34$ & 237 & \\
\hline No therapy & 16 & 11 & $6-16$ & 116 & \\
\hline \multicolumn{6}{|l|}{ Transplanted } \\
\hline Yes & 119 & 66 & $59-73$ & 195 & 0.02 \\
\hline No & 62 & 34 & $27-41$ & 248 & \\
\hline \multicolumn{6}{|c|}{ Explanted pathology } \\
\hline $\begin{array}{l}\text { Necrotic } \\
\text { tissue }\end{array}$ & 19 & 20 & $12-28$ & 207 & 0.59 \\
\hline $\begin{array}{l}\text { Residual } \\
\text { tumor }\end{array}$ & 75 & 80 & $71-88$ & 205 & \\
\hline $\begin{array}{l}\text { MELD-Na } \\
\text { score }\end{array}$ & 10 & $9-15$ & - & - & - \\
\hline Days in WL & $206^{*}$ & $50-291^{a}$ & - & - & - \\
\hline
\end{tabular}

NASH - nonalcoholic steatohepatitis, WL - waiting list, ${ }^{*}$ median, ${ }^{a}$ interquartile range. Missing values are not included

as coronary artery disease, hypertension, diabetes, chronic obstructive pulmonary disease, chronic kidney failure, and symptoms of portal hypertension. Patients were classified as within the Milan criteria (single nodule $>2 \mathrm{~cm}$ and $<5 \mathrm{~cm}$, or 3 nodules each $\leq 3 \mathrm{~cm}$ ) and outside of the Milan criteria, a designation which included patients with T0N0M0, T1N0M0, and T3N0M0. TNM classifications were registered at diagnosis. For comparative analysis of transplanted vs. non-transplanted patients (Table 2), the TON0M0 group was combined with the T1N0M0 group. LDTs were assigned according to decisions made by multidisciplinary teams. In addition, patients who received no therapy were included in the study as a comparative group. For the purpose of analysis, LDTs were categorized as trans-arterial therapies, including arterial chemoembolization (TACE) and Yttrium-90 (Y-90).
Thermal ablations included microwave ablation, radiofrequency, and electroporation (NanoKnife). Combined therapies were defined as the combination of any trans-arterial therapy and any ablation. The "no therapy" group was considered a control group. Our LDT data included the number cycles of each treatment (single cycle, multiple cycles, combined therapy, and non-therapy). MELD score was included as the calculation of the laboratory data. This study did not include documentation of the allocation MELD score, including the exception points assigned and updated by UNOS. The explanted pathology was reported according to the College of American Pathologists HCC cancer protocol (well, moderately, and poorly differentiated). Complete pathologic response (cPR) was defined as no viable tumor in the explanted liver pathology (necrotic tissue), and the presence of any tumor 
Table 2. Characteristics of transplanted vs. non-transplanted patients

\begin{tabular}{|c|c|c|c|c|c|c|}
\hline \multirow[t]{2}{*}{ Variables } & \multicolumn{3}{|c|}{ Transplanted $n=119$} & \multicolumn{3}{|c|}{ Not transplanted $n=62$} \\
\hline & $n$ & $\%$ or SD & $95 \% \mathrm{Cl}$ or $p$-value & $n$ & $\%$ or SD & $95 \% \mathrm{Cl}$ or $p$-value \\
\hline Total patients & 121 & 67 & & 60 & 33 & \\
\hline Age (mean) & 60.7 & 7.9 & & 58.5 & 7.2 & 0.08 \\
\hline \multicolumn{7}{|l|}{ Sex } \\
\hline Male & 89 & 74 & $66-82$ & 32 & 89 & $78-100$ \\
\hline Female & 32 & 26 & $18-34$ & 4 & 11 & $0-21$ \\
\hline \multicolumn{7}{|l|}{ Ethnicity } \\
\hline Hispanics & 59 & 52 & $42-61$ & 24 & 56 & $40-71$ \\
\hline Non-Hispanic Whites & 46 & 40 & $31-49$ & 18 & 42 & $26-57$ \\
\hline Others & 9 & 8 & $3-13$ & 1 & 2 & $0-7$ \\
\hline \multicolumn{7}{|l|}{ Etiology } \\
\hline Viral & 32 & 26 & $18-34$ & 33 & 55 & $42-68$ \\
\hline NASH & 16 & 13 & $7-20$ & 12 & 20 & $10-30$ \\
\hline Alcohol & 12 & 10 & $5-15$ & 13 & 21 & $11-32$ \\
\hline Unspecified & 56 & 46 & $37-55$ & 2 & 3 & $0-8$ \\
\hline Others & 5 & 4 & $1-8$ & & & \\
\hline \multicolumn{7}{|l|}{ Comorbidities } \\
\hline One & 57 & 67 & $57-77$ & 14 & 93 & $79-100$ \\
\hline Two & 21 & 25 & $15-34$ & 1 & 7 & $0-20$ \\
\hline Three & 7 & 8 & $2-14$ & - & - & - \\
\hline \multicolumn{7}{|l|}{ TNM classification } \\
\hline T1NOMO & 17 & 16 & $9-23$ & 5 & 9.8 & $1.3-18$ \\
\hline T2NOMO & 71 & 68 & $59-77$ & 29 & 8 & $43-71$ \\
\hline T3NOMO & 17 & 16 & $9-23$ & 17 & 33 & $20-47$ \\
\hline \multicolumn{7}{|l|}{ Milan criteria } \\
\hline Within Milan criteria & 71 & 68 & $59-77$ & 29 & 57 & $43-71$ \\
\hline Outside of Milan criteria & 34 & 22 & $23-41$ & 22 & 43 & $29-57$ \\
\hline \multicolumn{7}{|l|}{ Therapy } \\
\hline Intra-arterial & 49 & 54 & $43-64$ & 25 & 46 & $33-60$ \\
\hline Ablations & 13 & 14 & $7-22$ & 3 & 6 & $0-12$ \\
\hline Combined therapy & 22 & 24 & $15-33$ & 17 & 31 & $19-44$ \\
\hline No therapy & 7 & 8 & $2-13.2$ & 9 & 16 & $6.3-27$ \\
\hline \multicolumn{7}{|l|}{ Number of cycles } \\
\hline Single cycle & 41 & 45 & $35-55$ & 11 & 20 & $9-31$ \\
\hline Multiple cycles & 21 & 23 & $14-32$ & 17 & 31 & $17-44$ \\
\hline Combined therapy & 22 & 25 & $15-33$ & 17 & 31 & $17-44$ \\
\hline No therapy & 7 & 8 & $2-13.2$ & 9 & 17 & $6-27$ \\
\hline \multicolumn{7}{|l|}{ Explanted pathology } \\
\hline Necrotic tissue & 19 & 20 & $12-28$ & - & - & - \\
\hline Residual tumor & 75 & 80 & $72-88$ & - & - & - \\
\hline Median days WL & $195^{*}$ & $94-263^{a}$ & & $248^{*}$ & $127-490^{\mathrm{a}}$ & $0.02 * *$ \\
\hline
\end{tabular}

NASH - nonalcoholic steatohepatitis, WL - waiting list, * ${ }^{*}$ edian, ainterquartile range, ${ }^{* *} p$ value of the medians. Missing values are not included 
grade differentiation (well, moderately, and poorly differentiated) was classified as no response to LDT despite the presence of some necrotic tissue.

\section{Statistical analyses}

Demographic characteristics of the study population are shown as frequencies and percentages with 95\% confidence intervals (CIs). In some comparative analyses, CIs were used instead of $p$ values to evaluate statistical significance to facilitate interpretation of the results. Continuous variables are described as means and medians with SD or interquartile range (IQR) if the variable does not follow a normal distribution (e.g., days on waiting list, MELD score). The median time on the waiting list was compared using the Wilcoxon rank-sum test or Mann-Whitney $U$ test. Survival analysis was used to examine the time to transplant and factors that influenced that time. Kaplan-Meier estimates were used to determine the mean survival time to receive a liver transplant. Cox proportional-hazards ratios (HRs) were used to test and quantify the association between predictors (age at HCC diagnosis, sex, ethnicity, Milan criteria, TNM classification, LDTs, and MELD score) and waiting list time. Variable selection for the Cox proportional hazards model retained factors with $p<0.25$. Statistical analyses were performed using SAS 9.4, and the significance level was set at $p<0.05$. To build the Cox proportional HR model, missing information was handled with list-wise deletion by the SAS software.

\section{Results}

\section{Study population and median time on waiting list}

The characteristics of the study population are summarized in Table 1 . The mean age at HCC diagnosis was 60 years. HCC affected more men than women ( $77 \%$ vs. $23 \%$ ), and $53 \%$ of our study population was of Hispanic ethnicity. In the study population, $71 \%$ of patients had one comorbidity, and $29 \%$ had two or more comorbidities. The main etiologic agent for HCC was viral infection, represented in most cases by hepatitis C virus (HCV). One hundred nineteen (119/181, $66 \%)$ of the patients underwent transplantation, and $100 / 154(64 \%)$ patients were classified within the Milan criteria and as stage T2N0M0. The median waiting list time (IQR) was 206 (50-291) days. The median time on the waiting list was lower for men than for women (191 vs. 236 days, $p=0.0093$ ), for transplanted patients vs. not transplanted (195 vs. 248 days, $p=0.02$ ), and although not statistically significant, for patients un- der no therapy prior to LT compared with those under other therapies, such as combined therapy (116 vs. 237 days, $p=0.058$ ).

\section{Liver-directed therapies in the study population}

As shown in Table 1, eighty nine percent of patients (129/145) underwent LDT, as documented in their medical records. Seventy-four (51\%) patients received intra-arterial therapy, 70 patients received trans-arterial chemoembolization (TACE), and four patients received Y-90. Sixteen patients (11\%) were submitted to ablative therapies, and 39 patients (27\%) received combined therapies (any intra-arterial therapy associated with ablation). Additionally, 16 patients (11\%) did not receive therapy prior to liver transplant.

\section{Transplanted vs. non-transplanted patients}

Sixty-six percent of the patients underwent transplantation. The characteristics of transplanted and non-transplanted patients are shown in Table 2. Age, sex, ethnicity, Milan criteria, LDT types, and waiting list times were similar between the two groups. Pathologic study of the explanted liver reported complete pathologic response (necrotic tissue and non-viable tumor) in 20\% (95\% CI: $12-28 \%$ ) of transplanted patients, and $80 \%$ (95\% CI: 71-88\%) of patients had some type of viable tumor with different degrees of differentiation. LDTs prior to LT (intra-arterial, ablative, combined, and no therapy) were allotted in similar proportions in transplanted and non-transplanted patients $(p=0.121)$. However, single-cycle therapies comprised a bigger proportion of the therapies in transplanted patients when compared with non-transplanted patients ( $45 \%$ vs. $20 \%$ [ $95 \%$ CI: $34-55 \%$ vs. $9-31 \%]$ ).

In non-transplanted patients, reasons for dropout from the waiting list included tumor progression (50\% of patients), deterioration of health status (43\%), death during the waiting time (5\%), and other reasons (2\%).

\section{Effects of variables on transplant and waiting list time}

Kaplan-Meier analysis revealed the probability of undergoing transplantation at any specific point in time for the study population (Fig. 1). The median time for undergoing transplantation (number of days in the waiting list when $50 \%$ of the population received a liver transplant) was 218 days (95\% CI: 195-235 days). Twenty-five percent of the population had undergone transplantation by day 106 (95\% CI: 90-167), and $75 \%$ of the population had undergone transplanta- 
tion by day 442 (95\% CI: 287-644) (Fig. 1). The median survival times for women and men (when 50\% of women/men had undergone transplantation) were 236 days (95\% CI: 186-333 days) and 205 days (95\% CI: 189-222 days), respectively (log-rank test $p$ value $=0.057)($ Fig. 2$)$

Table 3 shows the effect of each independent variable on liver transplant probability and waiting list time. Calculation of unadjusted HRs indicated that age was the only variable significantly associated with the probability of receiving a transplant (HR: 1.03, $p=0.02$ ). A multivariable model revealed several factors associated with probability of undergoing transplantation. The HR, or probability of undergoing transplantation at any point in time, was 3.1 times greater for men than for women $(p=0.002)$. However, patients who received no therapy had a 5-fold higher probability of undergoing transplantation than patients under arterial LDT (HR [95\% CI]: 5 [1.2, 20], $p=0.02)$. Patients under combined LDT displayed a $70 \%$ reduced probability of transplantation compared to patients who received arterial LDTs (HR [95\% $\mathrm{CI}]: 0.3[0.1-0.6], p=0.0009)$. All of these effects were determined after adjusting for confounders, such as age, sex, etiology, comorbidities, TNM, and therapies (Table 4 and Fig. 3).

\section{Discussion}

Since its implementation in 2002, the MELD score has been the primary tool used to prioritize patients awaiting liver transplantation [14]. In this study, the MELD score was not used in the final Cox proportional-hazards model due to the lack of significance (small sample size) found in the univariate analysis (Table 3 ). Research has identified other factors that play a role in organ allocation and waiting list time. These factors include the MELD exception points, health status of the recipient, $\mathrm{ABO}$ blood type, transjugular intrahepatic portosystemic shunt placement, transplant region, ethnicity, and primary source of payment [14]. Our study showed that women and non-transplanted patients experienced longer waiting list times. Although not statistically significant $(p=0.058)$, patients under combined LDT also experienced longer waiting list times. In this analysis, the median time on the waiting list for the period 2014-2019 was 206 days (IQR: 50-291). According to OPTN, the median waiting times for deceased donor transplant in region 3 in patients with a MELD score < 15 during the periods 2003-2006 and 2011-2014 were 227 days (IQR: 185-286) and 222 days (IQR: 198-244), respectively [15]. In Trieu's analysis of the ONUS database, patients living in region 3

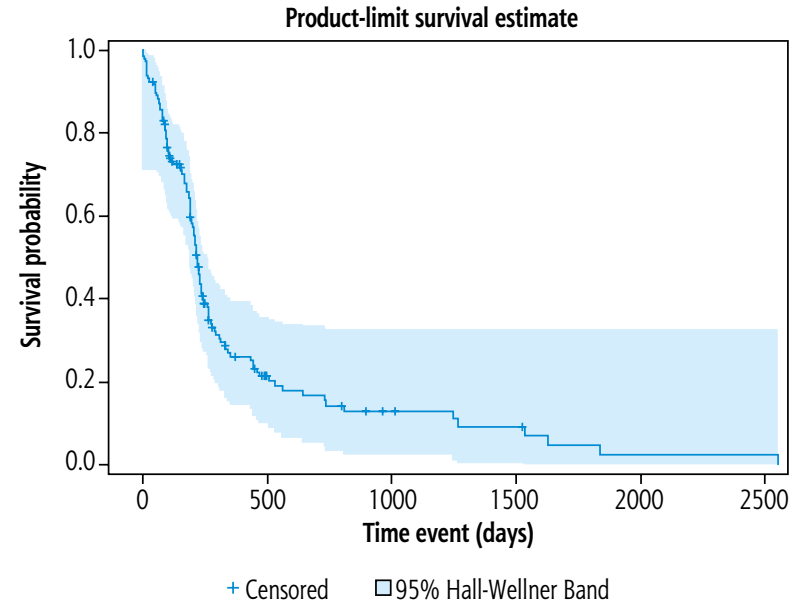

Fig. 1. Survival overall probability of undergoing transplantation, and confidence interval, for patients with hepatocellular carcinoma ( $\mathrm{HCC}$ ) in a single center

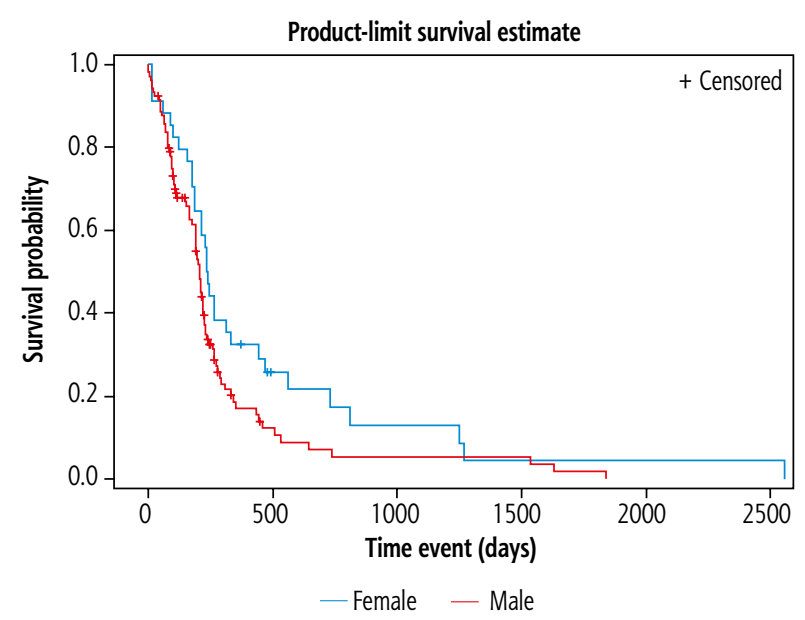

Fig. 2. Survival probability of undergoing transplantation by gender

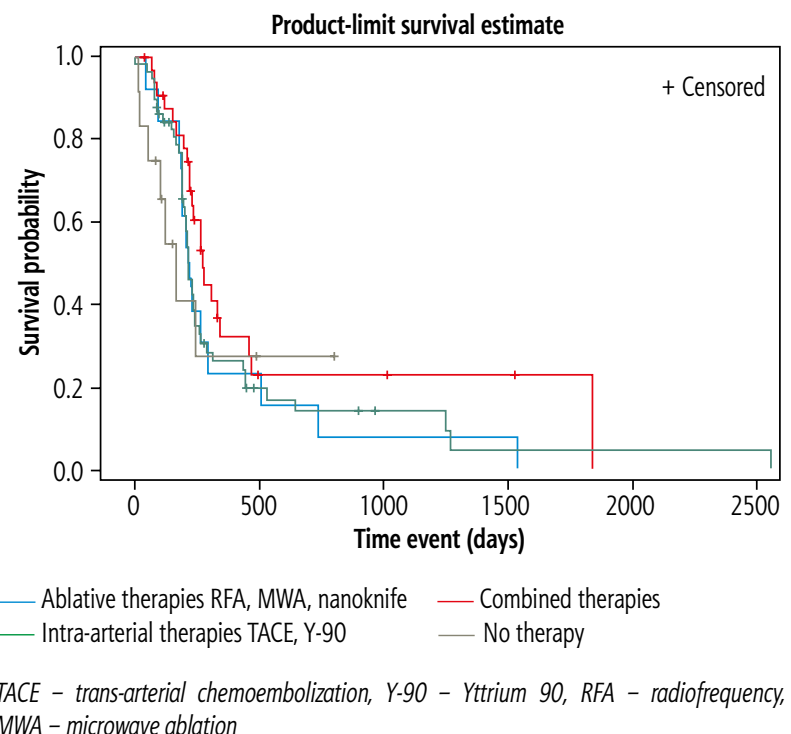

- microwave ablation

Fig. 3. Survival probability of undergoing transplantation by treatment 
Table 3. Crude hazard ratio (HR) for undergoing transplantation

\begin{tabular}{|c|c|c|c|}
\hline Variables & HR & $95 \% \mathrm{Cl}$ & $P$-value \\
\hline Age & 1.03 & $1.0-1.05$ & 0.02 \\
\hline \multicolumn{4}{|l|}{ Sex } \\
\hline Male & 1.5 & $0.98-2.3$ & 0.06 \\
\hline Female & Ref & & \\
\hline \multicolumn{4}{|l|}{ Ethnicity } \\
\hline Hispanics & Ref & & \\
\hline Non-Hispanic Whites & 0.98 & $0.7-1.5$ & 0.93 \\
\hline Others & 0.75 & $0.4-1.5$ & 0.43 \\
\hline \multicolumn{4}{|l|}{ Etiology } \\
\hline Viral & Ref & & \\
\hline NASH & 1.2 & $0.7-25$ & 0.38 \\
\hline Alcohol & 0.9 & $0.5-1.8$ & 0.74 \\
\hline Unspecified & 1.7 & $1.1-2.7$ & 0.02 \\
\hline Others & 0.9 & $0.4-2.4$ & 0.88 \\
\hline \multicolumn{4}{|l|}{ Comorbidities } \\
\hline One & Ref & & \\
\hline Two & 1.2 & $0.7-2.0$ & 0.56 \\
\hline Three & 1.8 & $0.8-3.9$ & 1.16 \\
\hline \multicolumn{4}{|l|}{ TNM classification } \\
\hline T1N0M0 & Ref & & \\
\hline T2NOMO & 0.7 & $0.4-1.2$ & 0.23 \\
\hline T3NOMO & 0.5 & $0.3-1.1$ & 0.08 \\
\hline \multicolumn{4}{|l|}{ Milan criteria } \\
\hline Within Milan criteria & Ref & & \\
\hline $\begin{array}{l}\text { Outside of Milan } \\
\text { criteria }\end{array}$ & 1 & $0.7-1.5$ & 0.98 \\
\hline \multicolumn{4}{|l|}{ Therapy } \\
\hline Intra-arterial & Ref & & \\
\hline Ablations & 1.1 & $0.6-2.0$ & 0.79 \\
\hline Combined therapy & 0.7 & $0.4-1.1$ & 0.13 \\
\hline No therapy & 1.2 & $0.5-2.7$ & 0.65 \\
\hline \multicolumn{4}{|l|}{ Number of cycles } \\
\hline Single cycle & Ref & & \\
\hline Multiple cycles & 0.8 & $0.5-1.5$ & 0.52 \\
\hline Combined therapy & 0.6 & $0.4-1.1$ & 0.08 \\
\hline No therapy & 1.1 & $0.5-2.5$ & 0.8 \\
\hline \multicolumn{4}{|l|}{ Explanted pathology } \\
\hline Necrotic tissue CPR & Ref & & \\
\hline Residual tumor & 0.74 & $0.4-1.2$ & 0.26 \\
\hline MELD score & 0.98 & $0.93-1.03$ & 0.37 \\
\hline
\end{tabular}

HR - hazard ratio, Cl - confidence interval, Ref - reference, NASH - nonalcoholic steatohepatitis, CPR - complete pathologic response
Table 4. Multifactorial model or adjusted hazard ratios for undergoing transplantation while on the waiting list

\begin{tabular}{|c|c|c|c|}
\hline Variables & HR & $95 \% \mathrm{Cl}$ & $P$-value \\
\hline Age & 0.99 & $0.95-1.04$ & 0.8 \\
\hline \multicolumn{4}{|l|}{ Sex } \\
\hline Male & 3.1 & $1.5-6.2$ & 0.002 \\
\hline Female & 1 & Ref & Ref \\
\hline \multicolumn{4}{|l|}{ Etiology } \\
\hline Viral & 1 & Ref & Ref \\
\hline NASH & 2.8 & $1.2-6.6$ & 0.018 \\
\hline Alcohol & 0.6 & $0.3-1.6$ & 0.63 \\
\hline Unspecified & 1.1 & $0.4-2.5$ & 0.9 \\
\hline Other & 1.8 & $0.3-8.8$ & 0.5 \\
\hline \multicolumn{4}{|l|}{ Comorbidities } \\
\hline One & 1 & Ref & Ref \\
\hline Two & 0.6 & $0.3-1.3$ & 0.2 \\
\hline Three & 0.4 & $0.08-2.5$ & 0.4 \\
\hline \multicolumn{4}{|l|}{ TNM classification } \\
\hline T1NOMO & 1 & Ref & Ref \\
\hline T2NOMO & 0.8 & $0.3-2.0$ & 0.7 \\
\hline T3NOMO & 1.1 & $0.4-3.2$ & 0.84 \\
\hline \multicolumn{4}{|l|}{ Therapy } \\
\hline Intra-arterial & 1 & Ref & Ref \\
\hline Ablatives & 0.6 & $0.23-1.5$ & 0.26 \\
\hline Combined therapy & 0.3 & $0.1-0.6$ & 0.0009 \\
\hline No therapy & 5 & $1.2-20$ & 0.02 \\
\hline
\end{tabular}

HR - hazard ratio, $\mathrm{Cl}$ - confidence interval, Ref - reference, NASH - nonalcoholic steatohepatitis

displayed the shortest waiting list times. This finding was due to the fact that in 2016, the number of livers available for transplant exceeded the number of registrations in that region [14].

Our results show that women exhibited a lower probability of undergoing transplantation as well as a longer waiting list time compared to men. Sex-based disparities in liver transplantation can be explained by several factors, including sex differences in the etiology of cirrhosis. Primary biliary cirrhosis and autoimmune hepatitis are more prevalent in women than men, but men are more likely than women to suffer from HCV and disease progression to cirrhosis [16]. Additionally, men display higher rates of alcohol abuse, but women exhibit increased susceptibility to the toxic effects of alcohol [16]. Hormonal factors can increase susceptibility to liver fibrogenesis after menopause in women affected by chronic liver disease [17]. Female biology, 
such as small muscle mass compared to that of men, contributes to lower creatinine levels and associated lower MELD scores compared to men [18]. The typically smaller size of females has been associated with a donor-recipient size mismatch, which increases waiting list time for females [19]. McElroy et al. found that women were less likely to be listed for liver transplantation due to higher active substance use when compared with men [20]. In summary, disease severity in women does not appear to be accurately reflected in their MELD score.

Age has been linked to an increased probability of undergoing transplantation. However, in our multivariate analysis, we did not observe an association between age and probability of liver transplant or waiting list time.

After covariate adjustment, patients who did not receive pre-transplant LDT exhibited a higher probability of undergoing transplantation compared with patients who received pre-transplant LDT. The same analysis revealed that during the first 250 days on the waiting list, patients who did not receive any LDT prior to liver transplant exhibited a higher probability of undergoing transplantation than patients who received LDT. After 250 days, none of the patients who did not receive LDT had undergone transplantation. This finding can be explained by the patients' health statuses: patients with worse health statuses likely had higher MELD scores, making liver transplant their only treatment option. This conclusion is supported by the observation that among transplanted patients with three comorbidities (seven subjects), five did not receive any therapy before the transplant. Factors associated with liver disease, such as severe ascites, are known to reduce liver transplant waiting list time [14], because these patients are given the highest priority [21]. Patients who required combined LDT were less likely to undergo transplantation at any point in time and remained on the waiting list longer when compared with patients receiving arterial LDT or no therapy. Gyori et al. observed non-significantly longer waiting time in patients receiving multimodal LDT (10.2 vs. 8 months, $p=0.07$ ) than in those receiving transarterial or ablative therapies. However, lesion size and tumor number were not significantly different between groups [22]. A combination of LDTs is expected to indicate aggressive disease, but LDT allows these patients to remain active on the liver transplant list, increasing their chances of receiving a transplant. These findings suggest that the severity of the tumor, not the type of therapy, increases the waiting list time, so use of multiple treatment modalities is necessary to achieve local control of the disease prior to LT.
This study reported an overall cPR of 20\% (95\% CI: $12-28 \%$ ) in explanted livers. This result was similar to DiNorcia's findings, where the overall cPR was 20.5\% [23]. The degree of tumor necrosis found on the explanted liver has been associated with recurrence and overall survival [23].

One strength of this study is that our findings highlight sex-based disparities in access to liver transplants. Women were three times less likely to undergo transplantation than men. However, we did not identify an explanation for this disparity, and thus a thorough analysis including economic factors, insurance coverage, and access to preventive care is necessary. One limitation of this single-center study is the number of individuals excluded due to missing data including MELD score exception points, which are assigned and updated by ONUS every 3 months. Additionally, the non-transplanted group displayed a high number of losses; thus, determining the precise dates of delisting and waiting times for this group was difficult. To handle these losses in our data, we used list-wise deletion in SAS software. In multivariate analyses, this approach results in deletion of an entire observation, decreasing the sample size and statistical significance of the results. In retrospective studies, researchers cannot control exposure and outcome assessment, and they must rely on others for accurate recording keeping. For example, for the etiology variable, most patients were classified as having "unspecified etiology", reducing the accuracy of our association between etiologic factors and outcome.

\section{Conclusions}

In this study, time spent on the liver transplant waiting list was associated with gender and type of LDT. Liver-directed therapy was associated with a prolonged stay on the transplant waiting list, likely due to the presence of an aggressive liver tumor. However, LDTs allow the patient to remain active on the liver transplant list, increasing their chances of undergoing transplantation.

\section{Disclosure}

The authors declare no conflict of interest.

\section{References}

1. Yang A, Ju W, He X. Comparison between liver resection and liver transplantation on outcomes in patients with solitary hepatocellular carcinoma meeting UNOS criteria: a population-based study of the SEER database. Oncotarget 2017; 8: 97428-97438. 
2. UNOS. United Network for Organ Sharing. Waiting for your transplant: 2019, available from: https://transplantliving.org/ before-the-transplant/waiting-for-your-transplant/.

3. Kamath PS, Wiesner RH, Malinchoc M, et al. A model to predict survival in patients with end-stage liver disease. Hepatology 2001; 33: 464-470.

4. Fernandez K. Fewer liver transplant candidates will die on the wait list. Hep 2015, available from: https://www.hepmag.com/ article/liver-transplant-waiting-27230-1939679397.

5. Columbia Surgery. Liver transplant waiting list. Center for disease and transplantation: 2019, available from: https://columbiasurgery.org/liver/liver-transplant-waiting-list.

6. UW Health. University of Wisconsin-Madison. Median time to transplant for waitlist patients: Transplant 2016, available from: https://www.uwhealth.org/transplant/median-time-to-transplant-for-waitlist-patients/12008.

7. Goldberg DS, Olthoff KM. Standardizing MELD exceptions: current challenges and future directions. Curr Transplant Rep 2014; 1: 232-237.

8. OPTN/UNOS. Organ Procurement Transplantation Network/ UNOS. Policy Notice Modification to HCC Extension Criteria: 2018, available from: https://optn.transplant.hrsa.gov/media/2411/modification-to-hcc-auto-approvalcriteria_policy-notice.pdf.

9. Yao FY, Bass NM, Nikolai B, et al. A follow-up analysis of the pattern and predictors of dropout from the waiting list for liver transplantation in patients with hepatocellular carcinoma: implications for the current org. Liver Transpl 2003; 9: 684-692.

10. Galuppo R, McCall A, Gedaly R. The role of bridging therapy in hepatocellular carcinoma. Int J Hepatology 2013; 2013: 419302.

11. Clavien PA, Lesurtel M, Bossuyt PM, et al. Recommendations for liver transplantation for hepatocellular carcinoma: an international consensus conference report. Lancet Oncol 2012; 13 : e11-22.

12. Majno P, Giostra E, Menthe G. Management of hepatocellular carcinoma on the waiting list before liver transplantation: Time to control trials. Liver Transpl 2007; 13 (11 Suppl 2): 27-35.

13. Iezzi R, Pompili M, Posa A, et al. Combined loco regional treatment of patients with hepatocellular carcinoma: State of the art. World J Gastroenterol 2016; 22: 1935-1942.

14. Trieu JA, Bilal M, Hmoud B. Factors associated with waiting time on the liver transplant list: an analysis of the United Network for Organ Sharing (UNOS) database. Ann Gastroenterol 2018; 31: 84-89.

15. OPTN. Organ Procurement and Transplant Network. Regional Data: 2020, available from: https://optn.transplant.hrsa.gov/ data/view-data-reports/regional-data/\#.

16. Oloruntoba OO, Moyla CA. Gender-based disparities in access to and outcomes of liver transplantation. World J Hepatol 2015; 7: 460-467.

17. Bissell DM. Sex and hepatic fibrosis. Hepatology 1999; 29: 988989.

18. Cholongitas E, Marelli L, Kerry A, et al. Female liver transplant recipients with the same GFR as male recipients have lower MELD scores - a systematic bias. Am J Transplant 2007; 7: 685-692.

19. Mindikoglu AL, Emre SH, Magder LS. Impact of estimated liver volume and liver weight on gender disparity in liver transplantation. Liver Transpl 2013; 19: 89-95.

20. McElroy LM, Likhitsup A, Winder GS, et al. Gender disparities in patients with alcoholic disease evaluated for liver transplantation. Transplantation 2020; 104: 293-298.
21. Mazzaferro V. Squaring the circle of selection and allocation in liver transplantation for HCC. An adaptive approach. Hepatology 2016; 63: 1707-1717.

22. Gyori GP, Felsenreich DM, Silberhumer GR, et al. Multimodality locoregional treatment strategies for bridging HCC patients before liver transplantation. Eur Surg 2017; 49: 232-243.

23. DiNorcia J, Florman S, Haydel B, et al. Pathological response to pretransplant locoregional therapy is predictive of patient outcome after liver transplantation for hepatocellular carcinoma. Analysis for US multicenter HCC transplant consortium. Ann Surg 2020; 271: 616-624. 